\title{
Boundary Stabilization of a More General Kirchhoff-Type Beam Equation*
}

\author{
Jianwen Zhang, Danxia Wang" \\ Department of Mathematics, Taiyuan University of Technology, Taiyuan, China \\ Email: jianwen.z2008@163.com, "danxia.wang@163.com
}

Received July 12, 2012; revised August 12, 2012; accepted September 12, 2012

\begin{abstract}
Simultaneously, considering the viscous effect of material, damping of medium, geometrical nonlinearity, physical nonlinearity, we set up a more general equation of beam subjected to axial force and external load. We prove the existence and uniqueness of global solutions under non-linear boundary conditions which the model is added one damping mechanism at 1 end. What is more, we also prove the exponential decay property of the energy of above mentioned system.
\end{abstract}

Keywords: Kirchhoff-Type Beam; Non-Linear Boundary; Global Solutions; Exponential Decay

\section{Introduction}

The problem is based on the equation

$$
u_{t t}+u_{x x x x}-\left(\alpha+\beta \int_{0}^{l}\left|u_{x}(s, t)\right|^{2} \mathrm{~d} s\right) u_{x x}=0
$$

which was proposed by Woinowsky-krieger [1], as a model for vibrating beams with hinged ends. One of the first mathematical analysis for the equation

$$
u_{t t}+u_{x x x x}-M\left(\int_{0}^{l}\left|u_{x}\right|^{2} \mathrm{~d} x\right) u_{x x}=0
$$

was done by Ball [2], which was later extended to an abstract setting by defining a linear operator $A$ by Medeiros [3]. In [4], Tucsnak considered the above beam equation which clamped boundary and obtained the exponential decay of the energy when a damping of the type $a(x) u_{t}$ is effective near the boundary. In the same direction, Kouemon Patchen [5] obtained the exponential decay of the energy for above-equation when a nonlinear damping $g\left(u_{t}\right)$ was effective in $\Omega$. To [6] considered the above kirchhoff-type beam equation under non-linear boundary conditions

$$
\begin{gathered}
u(0, t)=u_{x}(0, t)=u_{x x}(l, t)=0 \\
u_{x x x}(l, t)-M\left(\int_{0}^{l}\left|u_{x}\right|^{2}\right) u_{x}(l, t)=f(u(l, t))+g\left(u_{t}(l, t)\right)
\end{gathered}
$$

which the model is clamped at $x=0$ and is supported $x=$

${ }^{*}$ This work is supported by Natural Science Foundation of Shanxi Provice, and the Natural Science Foundation for Young Scientists of Shanxi Province (2010011008, 2011021002-2).

\# Corresponding author. $l$. He proved the existence and decay rates of the solutions. A rather general kirchhoff-type beam equation

$$
\begin{aligned}
& u_{t t}+\alpha u_{x x x x}+\gamma u_{x x x x t} \\
& \left.-\left(\beta+k \int_{0}^{l} u_{x}^{2} \mathrm{~d} x+\sigma \int_{0}^{l} u_{x} u_{x t} \mathrm{~d} x\right)\right) u_{x x}+\delta u_{t}=0
\end{aligned}
$$

was set up by Ball [7], who presented the existence and uniqueness of solution under linear boundary conditions. However the global solution and exponential decay for the more general beam equation is open under nonlinear boundary conditions. In the present work, we are concerned with the existence and uniqueness of solutions and the exponential decay property of energy on the nonlinear beam equation with external load

$$
\begin{aligned}
& u_{t t}+u_{x x x x}+\mu u_{x x x x t}+\eta u_{t} \\
& -\left(M\left(\left\|u_{x}\right\|^{2}\right)+N\left(\int_{0}^{l} u_{x t} u_{x} \mathrm{~d} x\right)\right) u_{x x}=q(x, t)
\end{aligned}
$$

with nonlinear boundary conditions

$$
\begin{gathered}
u(0, t)=u_{x x}(0, t)=u_{x}(l, t)=0 \\
u_{x x x}(l, t)+\mu u_{x x x t}(l, t)=f(u(l, t))+g\left(u_{t}(l, t)\right)
\end{gathered}
$$

and initial conditions

$$
u(x, 0)=u^{0}(x) \text { and } u_{t}(x, 0)=u^{1}(x)
$$

\section{Definition and Assumptions}

In this paper, our analysis is based on the Sobolev spaces

$$
V=\left\{u \in H^{2}(0, l) \mid u(0)=u_{x}(l)=0\right\},
$$




$$
W=\left\{u \in V \cap H^{4}(0, l) \mid u_{x x}(0)=0\right\}
$$

espectively equipped with the norm $\|u\|_{V}=\left\|u_{x x}\right\|$ and $\|u\|_{W}=\left\|u_{x x}\right\|+\left\|u_{x x x x}\right\|$. We assume that $f, g: R \rightarrow R$ are continuously differentiable functions such that

$$
f(s) s \geq 0
$$

and

$$
f(s) s-2 \hat{f}(s) \geq 0, \forall s \in R
$$

where

$$
\begin{gathered}
\hat{f}(s)=\int_{0}^{s} f(z) \mathrm{d} z \text { and } \\
g(0)=0
\end{gathered}
$$

and $\quad(g(r)-g(s))(r-s) \geq \rho|r-s|^{2}, \forall r, s \in R$

for some $\rho>0$.

Assume that the functions $M(\cdot), N(\cdot) \in C^{1}[0, \infty)$ are non-negative functions and respectively satisfy

and

$$
\begin{gathered}
M(0)=0 \\
\hat{M}(s)=\int_{0}^{l} M(z) \mathrm{d} z \\
N(0)=0
\end{gathered}
$$

and

$$
N(s) s \geq 0
$$

\section{Existence and Uniqueness of Global Solutions}

Now we come to the following conclusions of the existence and uniqueness of global solutions.

Theorem 1. Assume that the assumptions of (5)-(8) and $q(x, t) \in L^{2}\left([0, \infty) ; L^{2}(0, l)\right)$ hold. Then for any $u^{0}, u^{1} \in W$ satisfying the compatibility condition

$$
u_{x x x}^{0}(l)+\mu u_{x x x}^{1}(l)=f\left(u^{0}(l)\right)+g\left(u^{1}(l)\right)
$$

There exists a function $u$ satisfying (1)-(4) such that

$$
u \in L^{2}(0, \infty ; W) \cap C^{0}([0, \infty) ; V) \cap W^{2, \infty}\left(0, \infty ; L^{2}(0, l)\right)
$$

Proof. Let us solve the variational problem associated with (1)-(4), which is given by: find $u(t) \in W$ such that

$$
\begin{aligned}
& \int_{0}^{l} u_{t t} \omega \mathrm{d} x+\int_{0}^{l} u_{x x} \omega_{x x} \mathrm{~d} x+\mu \int_{0}^{l} u_{x x t} \omega_{x x} \mathrm{~d} x+\eta \int_{0}^{l} u_{t} \omega \mathrm{d} x \\
& +\left[M\left(\left\|u_{x}\right\|^{2}\right)+N\left(\int_{0}^{l} u_{x} u_{x t} \mathrm{~d} x\right)\right] \int_{0}^{l} u_{x} \omega_{x} \mathrm{~d} x \\
& +\left[f(u(l, t))+g\left(u_{t}(l, t)\right)\right] \omega(l) \\
= & \int_{0}^{l} q(x, t) \omega \mathrm{d} x
\end{aligned}
$$

for all $\omega \in V$. Let $\left\{\omega^{j}\right\}$ be a complete orthogonal system of $W$. For each $m \in N$, let us put

$$
W^{m}=\operatorname{span}\left\{\omega^{1}, \omega^{2}, \cdots, \omega^{m}\right\} .
$$

We search for a function

$$
u^{m}(t)=\sum_{j=1}^{m} a^{j}(t) \omega^{j}
$$

where $a^{j}(t)$ is a unknown function such that for any $\omega \in W^{m}$, and it satisfies the approximating equation

$$
\begin{aligned}
& \int_{0}^{l} u_{t t}^{m} \omega \mathrm{d} x+\int_{0}^{l} u_{x x}^{m} \omega_{x x} \mathrm{~d} x+\mu \int_{0}^{l} u_{x x t}^{m} \omega_{x x} \mathrm{~d} x+\eta \int_{0}^{l} u_{t}^{m} \omega \mathrm{d} x \\
& +\left[M\left(\left\|u_{x}^{m}\right\|^{2}\right)+N\left(\int_{0}^{l} u_{x}^{m} u_{x t}^{m} \mathrm{~d} x\right)\right] \int_{0}^{l} u_{x}^{m} \omega_{x} \mathrm{~d} x \\
& +\left[f\left(u^{m}(l, t)\right)+g\left(u_{t}^{m}(l, t)\right)\right] \omega(l) \\
& =\int_{0}^{l} q(x, t) \omega \mathrm{d} x
\end{aligned}
$$

with the initial conditions

$$
u^{m}(0)=u^{0} \text { and } u_{t}^{m}(0)=u^{1}
$$

Thus (11) and (12) are equivalent to the Cauchy problem of ODES in the variable $t$, which is known to have a local solution $u^{m}(t)$ in an interval $\left[0, t_{m}\right)\left(t_{m}<T\right)$ for any given $T>0$.

Estimate 1. By integration of (11) over $[0, t]\left(t<t_{m}\right)$ with $\omega=u_{t}^{m}(t)$, we see that

$$
\begin{aligned}
& \left\|u_{t}^{m}\right\|^{2}+\left\|u_{x x}^{m}\right\|^{2}+2 \mu \int_{0}^{t}\left\|u_{x x t}^{m}\right\|^{2} \mathrm{~d} s+\hat{M}(z(t)) \\
& +2 \int_{0}^{t} N\left(z_{t}(s)\right) z_{t}(s) \mathrm{d} s+2 \int_{0}^{t} g\left(u_{t}^{m}(l, s)\right) u_{t}^{m}(l, s) \mathrm{d} s \\
& +2 \hat{f}\left(u^{m}(l, t)\right) \\
= & \left\|u_{t}^{m}(0)\right\|^{2}+\left\|u_{x x}^{m}(0)\right\|^{2}+\hat{M}(z(0)) \\
& +2 \hat{f}\left(u^{m}(l, 0)\right)+2 \int_{0}^{t} \int_{0}^{l} q(x, s) u_{t}^{m} \mathrm{~d} s
\end{aligned}
$$

where $\quad z(t)=\left\|u_{x}^{m}\right\|^{2}$ and $z_{t}(t)=\frac{1}{2} \frac{\mathrm{d}}{\mathrm{d} t}\left\|u_{x}^{m}\right\|^{2}$.

Considering that

$$
g\left(u_{t}^{m}(l, s)\right) u_{t}^{m}(l, s) \geq \rho\left|u_{t}^{m}(l, s)\right|^{2}>0,
$$

$N\left(z_{t}(s)\right) z_{t}(s)>0$ and the initial conditions, we get

$$
\left\|u_{t}^{m}\right\|^{2}-\int_{0}^{t}\left\|u_{t}^{m}(s)\right\|^{2} \mathrm{~d} s \leq C+\int_{0}^{t}\|q(x, s)\|^{2} \mathrm{~d} s .
$$

Using Gronwall inequality, we have $\left\|u_{t}^{m}\right\|^{2} \leq C e^{T}$. Then there exists a constant $M_{1}$ depending only on $T$ such that

$$
\left\|u_{t}^{m}\right\|^{2}+\left\|u_{x x}^{m}\right\|^{2} \leq M_{1} .
$$

for any $t \in[0, T]$ and for all $m \in N$.

In this paper, $C$ is a constant independent of $m, t$ and denotes different value in different mathematical expression.

Estimate 2. Integrating by parts (11) with $\omega=u_{t t}^{m}(0)$ and $t=0$, and considering the compatibility condition (3) we get 


$$
\begin{aligned}
&\left\|u_{t t}^{m}(0)\right\|^{2} \\
&= {\left[M(z(0))+N\left(z_{t}(0)\right)\right] \int_{0}^{l} u_{x x}^{m}(0) u_{t t}^{m}(0) \mathrm{d} x } \\
&- \mu \int_{0}^{l} u_{x x x x t}^{m}(0) u_{t t}^{m}(0) \mathrm{d} x-\int_{0}^{l} u_{x x x x}^{m}(0) u_{t t}^{m}(0) \mathrm{d} x \\
&-\eta \int_{0}^{l} u_{t}^{m}(0) u_{t t}^{m}(0) \mathrm{d} x+\int_{0}^{l} q(x, 0) u_{t t}^{m}(0) \mathrm{d} x \\
& \leq\left\|u_{t t}^{m}(0)\right\|\left\{\left[M(z(0))+N\left(z_{t}(0)\right)\right]\left\|u_{x x}^{m}(0)\right\|+\left\|u_{x x x x}^{m}(0)\right\|\right. \\
&\left.\quad+\mu\left\|u_{x x x x t}^{m}(0)\right\|+\eta\left\|u_{t}^{m}(0)\right\|+\|q(0)\|\right\}
\end{aligned}
$$

Thus there exists a positive constant $M_{2}$ such that

$$
\left\|u_{t t}^{m}(0)\right\| \leq M_{2}, \forall m \in N .
$$

Estimate 3. Let us fix $t, \xi>0$ such that $\xi<T-t$. Tak-

$$
I_{1}=\int_{0}^{l}\left\{\left[M(z(t+\xi))+N\left(z_{t}(t+\xi)\right)\right] u_{x}^{m}(t+\xi)-\left[M(z(t))+N\left(z_{t}(t)\right)\right] u_{x}^{m}(t)\right\}\left(u_{x t}^{m}(t+\xi)-u_{x t}^{m}(t)\right) \mathrm{d} x .
$$

Let us estimate $\left|I_{1}\right|$. Since

$$
u^{m}(0, t)=u_{x}^{m}(l, t)=u_{x x}^{m}(0, t)=0,
$$

we have

$$
\left\|u^{m}\right\|_{\infty} \leq \sqrt{l}\left\|u_{x}^{m}\right\|,\left\|u_{x}^{m}\right\|_{\infty} \leq \sqrt{l}\left\|u_{x x}^{m}\right\|,\left\|u_{x}^{m}\right\| \leq l\left\|u_{x x}^{m}\right\|
$$

Noting that $\Delta M_{1}=M\left(z(t+\xi)-M(z(t))\right.$ and $\Delta M_{2}=$ $N\left(z_{t}(t+\xi)\right)-N\left(z_{t}(t)\right)$, then integrating by parts we have

$$
\begin{aligned}
\left|I_{1}\right|= & \mid\left[M(z(t+\xi))+N\left(z_{t}(t+\xi)\right)\right] \\
& \times \int_{0}^{l}\left(u_{x x}^{m}(t+\xi)-u_{x x}^{m}(t)\right)\left(u_{t}^{m}(t+\xi)-u_{t}^{m}(t)\right) \mathrm{d} x \\
& +\left(\Delta M_{1}+\Delta M_{2}\right) \int_{0}^{l} u_{x x}^{m}(t)\left(u_{t}^{m}(t+\xi)-u_{t}^{m}(t)\right) \mathrm{d} x
\end{aligned}
$$

Since $M(\cdot) \in C^{1}[0, \infty)$, by the Mean value theorem, from estimates 1 and (16) we have

$$
\begin{aligned}
\left|\Delta M_{1}\right| & =\left|M^{\prime}\left(\eta_{1}\right)\left(\left\|u_{x}^{m}(t+\xi)\right\|^{2}-\left\|u_{x}^{m}(t)\right\|^{2}\right)\right| \\
& \leq C\left\|u_{x x}^{m}(t+\xi)-u_{x x}^{m}(t)\right\|
\end{aligned}
$$

where $\eta_{1}$ is between $\left\|u_{x}^{m}(t+\xi)\right\|^{2}$ and $\left\|u_{x}^{m}(t)\right\|^{2}$.

By the Mean value theorem, we also have

$$
\left|\Delta M_{2}\right| \leq C\left\|u_{x x}^{m}(t+\xi)-u_{x x}^{m}(t)\right\|+C\left\|u_{t}^{m}(t+\xi)-u_{t}^{m}(t)\right\|
$$

Considering that $M(z(t+\xi) \leq C$ and $N(z(t+\xi) \leq C$, we conclude that there exists constants $k_{1}>0$ and $k_{2}>0$ such that

$$
\left|I_{1}\right| \leq k_{1}\left\|u_{x x}^{m}(t+\xi)-u_{x x}^{m}(t)\right\|^{2}+k_{2}\left\|u_{t}^{m}(t+\xi)-u_{t}^{m}(t)\right\|^{2}
$$

A argument for $f$ yields

$$
\begin{aligned}
& \left|f\left(u_{t}^{m}(l, t+\xi)\right)-f\left(u_{t}^{m}(l, t)\right)\right|\left|u_{t}^{m}(l, t+\xi)-u_{t}^{m}(l, t)\right| \\
\leq & k_{3} \| u_{x x}^{m}(t+\xi)-\left.u_{x x}^{m}(t)\right|^{2}+\rho\left|u_{t}^{m}(l, t+\xi)-u_{t}^{m}(l, t)\right|^{2}
\end{aligned}
$$

ing the difference of (11) with $t=t+\xi$ and $t=t$, and replacing $\omega$ by $u_{t}^{m}(t+\xi)-u_{t}^{m}(t)$, we get

$$
\begin{aligned}
& \frac{1}{2} \frac{\mathrm{d}}{\mathrm{d} t}\left[\left\|u_{t}^{m}(t+\xi)-u_{t}^{m}(t)\right\|^{2}+\left\|u_{x x}^{m}(t+\xi)-u_{x x}^{m}(t)\right\|^{2}\right] \\
& +\mu\left\|u_{x x t}^{m}(t+\xi)-u_{x x t}^{m}(t)\right\|^{2}+\eta\left\|u_{t}^{m}(t+\xi)-u_{t}^{m}(t)\right\|^{2} \\
& +\left[f\left(u^{m}(l, t+\xi)\right)-f\left(u^{m}(l, t)\right)\right]\left(u_{t}^{m}(l, t+\xi)-u_{t}^{m}(l, t)\right) \\
& +\left[g\left(u_{t}^{m}(l, t+\xi)\right)-g\left(\left(u_{t}^{m}(l, t)\right)\right]\left(u_{t}^{m}(l, t+\xi)-u_{t}^{m}(l, t)\right)\right. \\
& +I_{1} \\
& =\int_{0}^{l}(q(t+\xi)-q(t))\left(u_{t}^{m}(t+\xi)-u_{t}^{m}(t)\right) \mathrm{d} x
\end{aligned}
$$

where

where $k_{3}>0$ is a constant. Putting

$$
\phi_{m}(t, \xi)=\left\|u_{x x}^{m}(t+\xi)-u_{x x}^{m}(t)\right\|^{2}+\left\|u_{t}^{m}(t+\xi)-u_{t}^{m}(t)\right\|^{2}
$$

and taking into account of (17)-(18) and the assumptions of $g$, we deduce from (15) that

$$
\frac{\mathrm{d}}{\mathrm{d} t} \phi_{m}(t, \xi) \leq k_{4} \varphi_{m}(t, \xi), \forall t \in(0, T)
$$

where $k_{4}=\max \left\{k_{1}+k_{3}, k_{2}\right\}$. Therefore

$$
\phi_{m}(t, \xi) \leq \phi_{m}(0, \xi) e^{k_{4} T}
$$

Dividing the above inequality by $\xi^{2}$ and letting $\xi \rightarrow 0$ gives

$$
\left\|u_{x x t}^{m}(t)\right\|^{2}+\left\|u_{t t}^{m}\right\|^{2} \leq\left(\left\|u_{x x t}^{m}(0)\right\|^{2}+\left\|u_{t t}^{m}(0)\right\|^{2}\right) e^{k_{4} T} .
$$

From estimate 2 we find a constant $M_{3}>0$ such that

$$
\left\|u_{x x t}^{m}(t)\right\|^{2}+\left\|u_{t t}^{m}\right\|^{2} \leq M_{3}, \forall m \in N, \forall t \in[0, T] .
$$

With the estimates 1 - 3 we can use Lions-Aubin Lemma to get the necessary compactness in order to pass (11) to the limit. Then it is a matter of routine to conclude the existence of the global solution in $[0, T]$.

Theorem 2. The solution $u(t)$ of theorem 1 is unique.

Proof. Let $u, v$ be two solutions of (1)-(4) with the same initial data. Then writing $p=u-v$, putting $\omega=p_{t}$ in (10) and using mean value theorem, chauchy-schwarz inequality and Gronwall inequality, we may get $p=0$. Thus $u=v$.

\section{The Exponential DECAY of the Energy of System}

In order to establish our decay result, we define the energy of the system by 


$$
\begin{aligned}
E(t)= & \frac{1}{2}\left\|u_{t}(t)\right\|^{2}+\frac{1}{2}\left\|u_{x x}(t)\right\|^{2} \\
& +\frac{1}{2} \hat{M}\left(\left\|u_{x}(t)\right\|^{2}\right)+\hat{f}(u(l, t))
\end{aligned}
$$

where $\hat{M}(s)=\int_{0}^{s} M(z) \mathrm{d} z$. We have

Theorem 3. Let $u(t)$ be the solution given by theorem 1 as $q(x, t)=0$ and $g=0$. And assume that $N(s) s \geq 0$ and $f(s) s \geq 0$. Then there exist constants $\lambda_{2}, \lambda_{4}>0$ and $\lambda_{3}<0$ such that $E(t) \leq \lambda_{2} E(0) e^{\lambda_{3} t}+\lambda_{4} t e^{\lambda_{3} t}$.

To prove Theorem 3 , we firstly introduce two lemmas.

Let us define $\quad \psi(t)=\int_{0}^{l} u u_{t} \mathrm{~d} x+\frac{\eta}{2} \int_{0}^{l} u^{2} \mathrm{~d} x$.

Then we have the following lemmas.

Lemma 1. Let $E_{\varepsilon}(t)=\mu E(t)+\varepsilon \psi(t)$. Then there exists a constant $k_{5}>0$ such that

$$
\left|E_{\varepsilon}(t)-\mu E(t)\right| \leq \varepsilon k_{5} E(t), \forall \varepsilon>0 .
$$

Proof. By $\|u\|_{\infty} \leq \sqrt{l}\left\|u_{x}\right\|, \quad\left\|u_{x}\right\|_{\infty} \leq \sqrt{l}\left\|u_{x x}\right\| \quad$ and $\|u\| \leq l\left\|u_{x x}\right\|$ there exists $k_{5}>0$ such that

$$
\begin{aligned}
\left|E_{\varepsilon}(t)-\mu E(t)\right| & =\varepsilon \int_{0}^{l} u u_{t} \mathrm{~d} x+\frac{\eta \varepsilon}{2} \int_{0}^{l} u^{2} \mathrm{~d} x \\
& \leq \frac{\varepsilon l^{2}}{2}\left\|u_{x x}\right\|^{2}+\frac{\varepsilon}{2}\left\|u_{t}\right\|^{2}+\frac{\eta \varepsilon l^{2}}{2}\left\|u_{x x}\right\|^{2} \\
& \leq \varepsilon k_{5} E(t)
\end{aligned}
$$

where $k_{5}=(1+\eta) l^{2}$.

Lemma 2. There exist constants $\lambda_{0}>0$ and $\lambda_{1}$ such that

$$
\frac{\mathrm{d}}{\mathrm{d} t} E_{\varepsilon}(t) \leq-2 \lambda_{0} E(t)+\lambda_{1} .
$$

Proof. Taking the inner product of (1) with $u_{t}$ and considering that $N(s) s \geq 0$, we have

$$
\frac{\mathrm{d}}{\mathrm{d} t} E(t) \leq-\mu\left\|u_{x x t}\right\|^{2}-\eta\left\|u_{t}\right\|^{2} .
$$

Taking the inner product of (1) with $u$, we have

$$
\begin{aligned}
\frac{\mathrm{d}}{\mathrm{d} t} \psi(t)= & \int_{0}^{l} u_{t}^{2} \mathrm{~d} x-\int_{0}^{l} u_{x x}^{2} \mathrm{~d} x-\mu \int_{0}^{1} u_{x x t} u_{x x} \mathrm{~d} x \\
& -\left(M\left(\left\|u_{x}\right\|^{2}\right)\right)+N\left(\int_{0}^{l} u_{x} u_{x t} \mathrm{~d} x\right) \int_{0}^{l} u_{x}^{2} d x \\
& -f(u(l, t)) u(l, t)
\end{aligned}
$$

Thus

$$
\begin{aligned}
\frac{\mathrm{d}}{\mathrm{d} t} E_{\varepsilon}(t) \leq & -\mu^{2}\left\|u_{x x t}\right\|^{2}-\varepsilon^{2} \int_{0}^{l} u_{x x}^{2} \mathrm{~d} x-\varepsilon \mu \int_{0}^{l} u_{x x t} u_{x x} d x \\
& -\left(\varepsilon-\varepsilon^{2}\right) \int_{0}^{l} u_{x x}^{2} \mathrm{~d} x-(\eta \mu-\varepsilon)\left\|u_{t}\right\|^{2} \\
& -\varepsilon M\left(\left\|u_{x}\right\|^{2}\right) \int_{0}^{l} u_{x}^{2} \mathrm{~d} x-\varepsilon N\left(\int_{0}^{l} u_{x} u_{x t} \mathrm{~d} x\right) \int_{0}^{l} u_{x}^{2} \mathrm{~d} x \\
& -\varepsilon f(u(l, t)) u(l, t)
\end{aligned}
$$

Set $\quad h\left(u_{x x t}\right)=-\mu^{2}\left\|u_{x x t}\right\|^{2}-\varepsilon^{2} \int_{0}^{l} u_{x x}^{2} \mathrm{~d} x-\varepsilon \mu \int_{0}^{l} u_{x x t} u_{x x} \mathrm{~d} x$

Since $\Delta=(\varepsilon \mu)^{2}\left(u_{x x}\right)^{2}-4 \mu^{2} \varepsilon^{2} u_{x x}^{2}=-3(\varepsilon \mu)^{2} u_{x x}^{2} \leq 0$, we have $h\left(u_{x x t}\right) \leq 0$. Therefore

$$
\begin{aligned}
\frac{\mathrm{d}}{\mathrm{d} t} E_{\varepsilon}(t) \leq & -\left(\varepsilon-\varepsilon^{2}\right) \int_{0}^{l} u_{x x}^{2} \mathrm{~d} x-(\eta \mu-\varepsilon)\left\|u_{t}\right\|^{2} \\
& -\varepsilon M\left(\left\|u_{x}\right\|^{2}\right) \times \int_{0}^{l} u_{x}^{2} \mathrm{~d} x-\varepsilon N\left(\int_{0}^{l} u_{x} u_{x t} \mathrm{~d} x\right) \int_{0}^{l} u_{x}^{2} \mathrm{~d} x . \\
& -\varepsilon f(u(l, t)) u(l, t)
\end{aligned}
$$

From the Mean value theorem, there exists a constant $\lambda_{1}$ such that

$$
-\varepsilon N\left(\int_{0}^{l} u_{x} u_{x t} \mathrm{~d} x\right) \int_{0}^{l} u_{x}^{2} \mathrm{~d} x \leq \lambda_{1} .
$$

On writing $\lambda_{0}=\min \left\{\eta \mu-\varepsilon, \varepsilon-\varepsilon^{2}, 2 \varepsilon\right\}$, we have

$$
\frac{\mathrm{d}}{\mathrm{d} t} E_{\varepsilon}(t) \leq-2 \lambda_{0} E(t)+\lambda_{1}
$$

The proof of theorem 3. From lemma 1, we have

$$
\left(\mu-\varepsilon k_{5}\right) E(t) \leq E_{\varepsilon}(t) \leq\left(\mu+\varepsilon k_{5}\right) E(t) .
$$

From Lemma 2, we have

$$
\frac{\mathrm{d}}{\mathrm{d} t} E_{\varepsilon}(t) \leq-2 \lambda_{0} E(t)+\lambda_{1} .
$$

Therefore

$$
\frac{\mathrm{d}}{\mathrm{d} t} E_{\varepsilon}(t) \leq-2 \lambda_{0}\left(\frac{1}{\mu+\varepsilon k_{5}}\right) E_{\varepsilon}(t)+\lambda_{1} .
$$

By Gronwall inequality and combing (21), we have

$$
\begin{aligned}
\left(\mu-\varepsilon k_{5}\right) E(t) & \leq\left[E_{\varepsilon}(0)+\lambda_{1} t\right] e^{\left(-2 \lambda_{0} \frac{1}{\mu+\varepsilon k_{5}} t\right)} \\
& \leq\left[\left(\mu+\varepsilon k_{5}\right) E(0)+\lambda_{1} t\right] e^{\left(-2 \lambda_{0} \frac{1}{\mu+\varepsilon k_{5}} t\right)} .
\end{aligned}
$$

Hence, for sufficiently small $\varepsilon>0$

$$
E(t) \leq \frac{\mu+\varepsilon k_{5}}{\mu-\varepsilon k_{5}} E(0) e^{\left(-2 \lambda_{0} \frac{1}{\mu+\varepsilon k_{5}} t\right)}+\frac{\lambda_{1}}{\mu-\varepsilon k_{5}} e^{\left(-2 \lambda_{0} \frac{1}{\mu+\varepsilon k_{5}} t\right)} .
$$

On writing $\quad \lambda_{2}=\frac{\mu+\varepsilon k_{5}}{\mu-\varepsilon k_{5}}, \lambda_{3}=\frac{-2 \lambda_{0}}{\mu+\varepsilon k_{5}}<0$

and

$$
\lambda_{4}=\frac{\lambda_{1}}{\mu-\varepsilon k_{5}},
$$

we have

$$
E(t) \leq \lambda_{2} E(0) e^{\lambda_{3} t}+\lambda_{4} t e^{\lambda_{3} t} .
$$

The proof of theorem 3 is now completed.

\section{REFERENCES}

[1] S. Woinowsky-Krieger, "The Effect of Axial Force on the 
Vibration of Hinged Bars," Journal of Applied Mechanics, Vol. 17, 1950, pp. 35-36.

[2] J. M. Ball, "Initial-Boundary Value Problems for an Extensible Beam," Journal of Mathematical Analysis and Applications, Vol. 42, No. 1, 1973, pp. 61-90. doi:10.1016/0022-247X(73)90121-2

[3] L. A. Mederios, "On a New Class of Nonlinear Wave Equations," Journal of Mathematical Analysis and Applications, Vol. 69, No. 1, 1979, pp. 252-262.

[4] M. Tucsnak, "Semi-Internal Stabilization for a Nonlinear Euler-Bernoulli Equation," Mathematical Methods in the Applied Sciences, Vol. 19, No. 11, 1996, pp. 897-907. doi:10.1002/(SICI)1099-1476(19960725)19:11<897::AID-
MMA801>3.0.CO;2-\#

[5] S. Kouemou Patcheu, "On a Global Solution and Asymptotic Behavior for the Generalized Damped Extensible Beam Equation," Journal of Differential Equations, Vol. 135, No. 2, 1997, pp. 299-314. doi:10.1006/jdeq.1996.3231

[6] F. M. To, "Boundary Stabilization for a Non-Linear Beam on Elastic Bearings," Mathematical Methods in the Applied Sciences, Vol. 24, No. 8, 2001, pp. 583-594. doi: $10.1002 / \mathrm{mma} .230$

[7] J. M. Ball, "Stability Theory for an Extensible Beam," Journal of Differential Equations, Vol. 14, No. 3, 1973, pp. 61-90. doi:10.1016/0022-0396(73)90056-9 